\title{
Aprendizaje con MOODLE
}

\section{Learning with MOODLE}

Marlene Rizo Rodríguez ${ }^{1}$

Recibido: 1 de diciembre de 2018, Aceptado: 6 de febrero de 2018

\section{RESUMEN}

La plataforma virtual MOODLE es un Sistema de Gestión de Aprendizaje en el que los docentes universitarios pueden diseñar y facilitar diferentes asignaturas, dado que posee una interfaz fácil y proporciona un conjunto de herramientas y ambientes que permiten desarrollar de forma colaborativa las actividades propuestas, por ende, los conocimientos, habilidades y destrezas que adquieren los estudiantes son significativos. En este ensayo se destacan conceptos básicos relacionados con este medio de enseñanza, características, herramientas, recursos; evaluación, así como una breve experiencia en la facilitación de la asignatura Informática Básica de forma virtual mediante este escenario.

Palabras claves: MOODLE; herramientas; recursos; informática básica.

\section{ABSTRACT}

The virtual platform MOODLE is a learning management system where the university teacher can design and teach different subjects since it has a user-friendly interface and provides a set of tools and environments that allow collaborative development of the proposed activities. Therefore, the knowledge, skills, and abilities acquired by students are significant. This essay highlights the basic concepts related to this teaching system, characteristics, tools, resources; evaluation and brief experience in the usage of the basic computing subject in a virtual approach through this scenario.

Keywords: MOODLE; tools; resources; basic computing.

1 Docente del Departamento de Ciencia, Tecnología y Salud, UNAN-Managua/FAREM-Estelí. Correo electrónico: mrrodriguez08@ yahoo.es

Copyright (c) 2018 Revista Multi-Ensayos.

(c) (1) (-) Este trabajo está licenciado bajo una Licencia Internacional Creative Commons 4.0 Atribución-NoComercial-Compartirlgual. 


\section{INTRODUCCIÓN}

Los seres humanos se han adaptado al uso de nuevas herramientas tecnológicas que cada día aparecen para auxiliarse en la realización desde las tareas cotidianas hasta las de profesionalización. En este contexto, las instituciones educativas, principalmente las universidades se están enfocando en ofrecer otras alternativas de aprendizaje además de la modalidad presencial, utilizando la plataforma virtual MOODLE para ofrecer carreras, cursos de educación continua, cursos de posgrado principalmente, entre otros.

\section{DESARROLLO}

La Universidad Nacional Autónoma de Nicaragua (UNAN-Managua) se ha caracterizado por estar a la vanguardia y hacer uso adecuado de las Tecnologías de la Información y la Comunicación (TIC). Por ello, en su Plan Estratégico 2015-2019 propone objetivos estratégicos en cada una de las funciones de Docencia, Investigación, Proyección y Extensión e Internacionalización, para alcanzarlos define estrategias y describe los resultados esperados. Éstas tienen como referente un Modelo Educativo que define directrices concretas para el ejercicio de las funciones universitarias que desarrolla la comunidad educativa, en el marco de la formación integral de los futuros profesionales.

En este contexto, la incorporación de TIC en el proceso de enseñanza-aprendizaje debe ser permanente. Dentro de la Función Docencia se estipula el siguiente objetivo estratégico que íntegramente dice: "Desarrollar la innovación pedagógica mediante la sistematización de la investigación y del quehacer educativo, en los procesos de enseñanza aprendizaje en cada uno de los niveles de formación que emprende la Universidad, que incorpore las TIC, actitudes creativas y emprendedoras". Para el cumplimiento del mismo propone como estrategia "Incorporación permanente de las Tecnologías de Información y Comunicación (TIC) en los procesos de enseñanza-aprendizaje según niveles de formación" (UNAN, Managua, 2015, pág. 22).

Es así que, todas las Facultades cuentan con sus aulas virtuales para desarrollar carreras completas, asignaturas de pregrado y posgrado, capacitaciones tanto al personal docente como no docente para el desarrollo de procesos de manera creativa e innovadora.

Además, es meritorio mencionar que se han hecho investigaciones que reflejan resultados positivos sobre estos escenarios de aprendizajes, una de ellas fue elaborada por Fonseca, Medrano y Orozco (2008) titulada "Impacto del uso de la Plataforma Virtual Moodle en la Carrera de Informática Educativa ofrecida por el Departamento de Informática Educativa de la Facultad de Educación e Idiomas, UNAN - Managua en la Modalidad de Profesionalización en el periodo 2008 - 2012".

Los resultados de este estudio demuestran que la Plataforma MOODLE responde a las necesidades educativas de los estudiantes de la modalidad de profesionalización e incide positivamente en el aspecto económico y en el acceso a la información de manera rápida, fácil y puntual; intercambian y refuerzan conocimientos, propiciando el aprendizaje colaborativo.

Por lo anterior, se puede afirmar que la UNAN-Managua con el uso de aulas virtuales para el proceso de enseñanza-aprendizaje, pone a la disposición de los interesados una alternativa adicional para lograr sus metas académicas de manera integral y de calidad. Asimismo, capacita a su personal para facilitar procesos de aprendizajes en línea, mediante la plataforma virtual MOODLE. 
Pero, ¿Qué es MOODLE?

MOODLE es un acrónimo en inglés de Module Object-Oriented Dynamic Learning Environment (Entorno de Aprendizaje Dinámico Modular Orientado a Objeto). Es una de las plataformas más usadas en la educación, es un centro de gestión de aprendizajes con enfoque constructivista permitiendo la comunicación entre los participantes y promoviendo el trabajo cooperativo.

Una de las principales características de MOODLE, radica en que es un software construido para el aprendizaje globalmente diseñado para soportar la enseñanza-aprendizaje, fácil de usar, gratuito, actualizado, flexible, seguro, escalable, robusto, basado en web, entre otras. Esto implica que las personas tienen la posibilidad para estudiar y aprender sin limitaciones de espacio y tiempo, basta con asumir el rol de estudiante virtual para hacer uso de los recursos y herramientas para el proceso de aprendizaje.

Para comprender mejor estas características, de acuerdo a lo que señala Murillo (2012) se describen a continuación:

\section{Construido para el aprendizaje. Mundialmente probado y de confianza}

Impulsando a decenas de miles de ambientes de aprendizaje global, MOODLE tiene la confianza de instituciones y organizaciones grandes y pequeñas. El número de usuarios de MOODLE a nivel mundial, de más de 79 millones de usuarios, entre usuarios académicos y empresariales, lo convierten en la plataforma de aprendizaje más ampliamente utilizada del mundo.

\section{Diseñado para soportar tanto la enseñanza como el aprendizaje}

Con más de 10 años de desarrollo guiado por la pedagogía de constructivismo social, MOODLE proporciona un conjunto poderoso de herramientas centradas en el estudiante y ambientes de aprendizaje colaborativo, que le dan poder, tanto a la enseñanza como al aprendizaje.

\section{Fácil de usar}

Una interfaz simple, que posee características de arrastrar y soltar, y recursos bien documentados, junto con mejoras continuas en usabilidad, hacen a MOODLE fácil de aprender y usar.

\section{Gratuito, sin cargos por licenciamiento}

MOODLE es proporcionado gratuitamente como programa de Código Abierto, bajo la Licencia Pública General GNU (GNU General Public License). Cualquier persona puede adaptar, extender o Modificar MOODLE, tanto para proyectos comerciales como no-comerciales, sin pago de cuotas por licenciamiento, y beneficiarse del costo/beneficio, flexibilidad y otras ventajas de usar MOODLE.

\section{Siempre actualizado}

La implementación de MOODLE en código abierto significa que MOODLE es continuamente revisado y mejorado, para adecuarse a las necesidades actuales y cambiantes de sus usuarios. 


\section{Altamente flexible y completamente personalizable}

Debido a que es código abierto, MOODLE puede ser personalizado en cualquier forma deseada, para adecuarlo a necesidades individuales. Su configuración modular y diseño interoperable les permite a los desarrolladores crear plugins e integrar aplicaciones externas para lograr funcionalidades específicas. Extienda lo que hace Moodle al usar plugins y complementos disponibles libremente, ilas posibilidades son ilimitadas!

\section{Escalable a cualquier tamaño}

Desde unos cuantos estudiantes hasta millones de usuarios, MOODLE puede escalarse para soportar las necesidades, tanto de clases pequeñas, como de grandes organizaciones, debido a su flexibilidad y escalabilidad, MOODLE ha sido adoptada para usarse en educación, negocios, organizaciones no lucrativas y contextos comunitarios.

\section{Robusto, seguro y privado}

Está comprometido con el resguardo de la seguridad de los datos y la privacidad del usuario, controles de seguridad que son constantemente actualizados, y habiendo implementado procesos del desarrollo de MOODLE y software para protección contra acceso no autorizado, pérdida de datos y mal uso, puede ser desplegado fácilmente en un servidor, o en una nube segura privada para un completo control.

\section{Puede usarse en cualquier momento, en cualquier lugar, en cualquier dispositivo}

MOODLE está basado en web, por lo que puede accederse a él desde cualquier lugar del mundo. Con una interfaz por defecto compatible con dispositivos móviles (que pronto será responsiva) y compatibilidad cruzada con diferentes navegadores de Internet, el contenido en la plataforma Moodle es fácilmente accesible y consistente a lo ancho de diferentes navegadores y dispositivos.

Como se puede apreciar, estas características y otras no mencionadas permiten que esta plataforma sea usada por pequeñas y grandes comunidades educativas. Además, proporciona herramientas que permiten al docente o facilitador implementarlas de acuerdo al nivel del curso o asignatura que facilitará de una manera fácil para que los estudiantes trabajen de forma individual y colaborativa.

Según Quiroz (2011), las herramientas disponibles en Moodle facilitan el aprendizaje de estudiantes. En tanto, a los docentes ayuda a crear cursos de calidad en línea, contempla módulos que implementan formas de relación entre los participantes, los materiales y las herramientas de comunicación integrados en un entorno común.

Entre las herramientas que pueden ser implementadas como estrategias pedagógicas en esta plataforma, están las siguientes:

Foros: Sirven para que tanto los docentes y estudiantes coloquen y discutan temas de interés en un curso, pueden participar las veces que deseen o las que se establezcan para realizar aportes del mismo con el fin de ampliar conocimientos y compartir experiencias.

Glosario: Docentes y estudiantes pueden crear el mismo. Es un diccionario que contiene definiciones de términos relacionados con las temáticas que se estén desarrollando. De esta manera se conoce el significado de nuevas palabras y por tanto se enriquece el vocabulario. 
Los recursos: Tienen la función principal de permitir que el docente incluya documentación relacionada con los temas a desarrollarse. De esta manera los estudiantes disponen de manuales, material de lectura, vídeos, link a otras páginas que amplíen información sobre un elemento específico para ayudar a entender de manera adecuada los temas y además que promueva aprendizajes y desarrollen habilidades.

Cuestionario: Permite la evaluación de los conocimientos que van adquiriendo los estudiantes. Los estudiantes pueden autoevaluar los aprendizajes adquiridos. Son fáciles de diseñar con diferentes tipos de preguntas. Es flexible porque se puede programar más de un intento para que los estudiantes puedan identificar sus respuestas no correctas y corregirlas.

Tareas: Como parte del cumplimiento del proceso de aprendizaje permite a estudiantes realizar las actividades propuestas por el docente y subir archivos de los trabajos realizados. El docente revisa, evalúa y retroalimenta las tareas, brinda comentarios y sugerencias a los estudiantes ya sea de manera individual o colectiva.

La consulta: Es una de las herramientas esenciales, ya que le permite al docente realizar preguntas a los estudiantes, para conocer sus puntos de vista. Es recomendable usar la opción de consulta anónima para tratar asuntos o contenidos que requieran privacidad.

La Wiki: Posibilita que docentes y estudiantes creen un documento de manera colaborativa sobre un tema de interés según la asignatura que se esté abordando. Inicia con una página principal y los participantes pueden agregar más páginas.

Además de las herramientas de Moodle, existe variedad de actividades lúdicas, tales como: juegos, la sopa de letras, el ahorcado; los cuales tienen propósitos educativos y deben ser planificados con el objetivo de alcanzar aprendizajes, tomando en cuenta el nivel de los participantes con que se esté trabajando.

También es importante mencionar los cambios que las nuevas tecnologías han implicado en la evaluación de los aprendizajes, Cabreiros (2011) citando a (Barberá 2006: 7-9) señala que éstos se refieren a la evaluación automática, la enciclopédica y la colaborativa. La primera es la más generalizada y conocida, puesto que se realiza a través de bases de datos que contienen bancos de preguntas que se presentan en diferentes formatos y que producen una rápida corrección de modo automático. La segunda descansa sobre la idea de trabajos específicos elaborados gracias a los contenidos que se pueden encontrar en internet. Por último, la evaluación colaborativa tiene en cuenta el trabajo realizado con otras personas mediante foros, debates y grupos de trabajo. (pág. 5)

Asimismo, cita a (Fontán 2004:3) para indicar que la evaluación de aprendizajes a través de la web, es única, permite una evaluación más global implicando a profesores, tutores, objetivos, contenidos, actividades, etc. Igualmente, el profesor deja de ser el único evaluador para presentarse las posibilidades de la autoevaluación o la evaluación entre pares. Además, dejan de valorarse sólo resultados y conocimientos para tenerse en cuenta tanto todo el proceso seguido como las actitudes y procedimientos. (pág. 5)

A continuación, una breve experiencia del proceso de aprendizaje de la asignatura de Informática Básica desarrollada mediante la plataforma virtual MOODLE en la FAREM Estelí, UNAN-Managua. 
Esta es la primera experiencia facilitando esta asignatura de manera virtual, debo confesar que al inicio hasta sentí que era un reto difícil, sin embargo, como recién había recibido dos cursos sobre Administración del Aprendizaje y Comunicación sobre Ambientes Virtuales Educativos, ambos impartidos por la Dirección de Educación a Distancia Virtual de la UNAN- Managua, me apropié de la metodología de manera inmediata y logré dirigir el proceso de aprendizaje, que aunque fue arduo, los resultados los considero exitosos, tanto para la facilitadora como para los participantes.

En el primer contacto que se tuvo con los estudiantes fue para motivarlos, entregar las credenciales: usuario y contraseña que les permitía el ingreso al aula virtual. Una vez familiarizados con la misma se explicaron las actividades que debían desarrollar en cada semana. Para ello se contaba con los recursos de aprendizajes, entre ellos: documentos bases para lectura, vídeos, link, imágenes, tutoriales; actividades a desarrollar definidas en cada semana con las orientaciones bien definidas y claras para que los estudiantes pudieran seguir las mismas sin dificultad y en el caso que las hubiese estaba a disposición el foro de consultas; en este espacio docente y estudiantes podían contestar para conducir a finalizar las tareas propuestas y de esta manera crear un ambiente de aprendizaje colaborativo.

Para dar seguimiento a las participaciones de los estudiantes el esfuerzo y dedicación se realizaba diario, en primer lugar, porque si habían inquietudes por parte de los estudiantes, poder responder inmediatamente para que avanzaran y en segundo lugar para revisar si las actividades cumplían con las orientaciones estipuladas; en caso de faltar alguna o varias de ellas se retroalimentaban y se daba la oportunidad para que los estudiantes corrigieran y que nuevamente actualizaran los archivos.

Es satisfactorio revisar de nuevo un archivo, una tarea actualizada y comprobar que los estudiantes atienden las sugerencias y que han logrado apropiarse más de conocimientos, habilidades y destrezas relacionadas con la informática.

En cuanto a la evaluación, cada actividad tiene sus propios criterios, el estudiante debía atenderlos para la obtención de la máxima calificación, ya sea de forma sumativa o formativa. En su mayoría los estudiantes incorporaron todos los criterios de evaluación en cada tarea asignada, sin embargo, algunos se conformaban con la evaluación obtenida inicialmente, ya que por alguna u otra razón no quisieron atender las sugerencias enviadas.

Esta experiencia de aprendizaje compartido fue enriquecedora, en vista que docente y estudiantes ampliamos nuestros conocimientos, lo mejor de todo fue poder concluir con éxito este proceso y saber que los estudiantes se llevan algo nuevo que les servirá en su vida académica, porque a lo mejor muchos de ellos van a seguir profesionalizándose y lo podrán hacer de manera virtual.

Por último, comparto reflexiones que realizaron los estudiantes al finalizar el curso, relacionadas con las siguientes interrogantes:

\section{¿Qué aprendí?}

"Aprendí muchos nuevos conocimientos que me ayudarán en el trascurso de mi carrera y como profesional, aprendí todo lo básico para utilizar el paquete de office que es esencial en todo trabajo", (Díaz R., 2018).

"Aprendí a utilizar el paquete de Microsoft office: Desde Word en la elaboración de documentos ordenados, inserción de citas según las normas APA; en Excel con la elaboración de tablas de contenidos, gráficos, inserción de fórmulas, aplicar formatos; en PowerPoint para la elaboración de presentaciones 
de mayor agrado para los receptores, el ingreso de imágenes, animaciones, transiciones, etcétera y también el uso de la plataforma virtual Moodle, el uso adecuado del computador, sus componentes, sistema operativo, internet y la web, entre otras cosas. (Gadea, 2018)

\section{¿Cómo lo aprendí?}

"Realizando las actividades indicadas, siguiendo las orientaciones, aplicando correcciones expuestas por el docente y siendo autodidacta", (Gadea, 2018).

“Lo aprendí gracias a la facilidad que tiene la profesora para explicarnos sus conocimientos y también la facilidad que tiene para desarrollar las temáticas que se presentaban en cada unidad. De igual manera, lo aprendido fue gracias a la plataforma porque en ella nos presentaban manuales para la realización de las actividades y los videos que se exponían", (Manzanares, 2018).

\section{¿Qué fortalezas desarrollé?}

"Planear mejor mi tiempo y mayor habilidad para usar las TIC", (Díaz A, 2018).

"Desarrollé la habilidad de manejar el teclado con más agilidad, insertar citas, el hábito de la lectura y sobre todo a analizar las indicaciones", (Aguilar, 2018).

\section{¿Dónde aplicaré las habilidades adquiridas?}

“Las aplicaré en las áreas estudiantiles y laborales para facilitar el trabajo, ahorrar tiempo y desarrollar nuevas ideas" (Castro, 2018).

"Las aplicaré en cada trabajo que haga como estudiante y futura maestra de Física-Matemática", (Morales, 2018).

\section{¿Qué debo mejorar?}

Aprendí mucho durante el desarrollo de esta plataforma virtual; sin embargo, debo mejorar en algunos aspectos del uso de hojas de cálculo. En mi carrera Contaduría Pública y Finanzas es necesario el buen uso de una hoja de cálculo, por lo tanto, es muy importante que aprenda a desarrollar otras fórmulas, es decir, que debo tomar un curso de Excel avanzado y así mejorar mis conocimientos. (Dávila, 2018). "Debo mejorar el dominio de esta herramienta para desarrollar un mejor trabajo y así alcanzar el conocimiento esperado y poder facilitarlo en las aulas de clase y en la vida cotidiana", (Rodríguez, 2018)

\section{CONCLUSIÓN}

La plataforma MOODLE es una de las más utilizadas por las instituciones educativas, en este caso, la FAREM Estelí, UNAN-Managua, hace uso de ellas para facilitar asignaturas de pregrado y posgrado, sus ventajas son grandes en comparación con las desventajas que pueden presentarse.

Los estudiantes se motivan al utilizar esta modalidad de aprendizaje, tienen la ventaja de tener disponible la información ya que es segura además que pueden acceder a sus contenidos a cualquier instante, Para el docente es muy útil contar con estas nuevas herramientas de tecnología de la información y comunicación, es importante, mencionar que no reemplazan el papel que desempeña el docente en el 
proceso de aprendizaje, por el contrario éste debe tener más disposición y vocación para el intercambio de conocimientos en esta modalidad virtual.

También, es necesario señalar que en esta plataforma los cursos son flexibles por el acceso a la diversidad de materiales, a mecanismos dinámicos de evaluación; asimismo que a los estudiantes les llega mucha información de retroalimentación. Todo esto apunta a alcanzar los objetivos de la asignatura de Informática Básica, asignatura de formación general para todas las carreras que se ofertan en la FAREM Estelí - UNAN Managua.

Finalizo agradeciendo la FAREM Estelí - UNAN Managua por la confianza depositada para facilitar la asignatura de Informática Básica en la plataforma MOODLE, fue una experiencia enriquecedora de conocimientos, también a los estudiantes por el trabajo constante, el esfuerzo y la dedicación que entregaron, aprendí mucho de ustedes, muchas gracias.

\section{BIBLIOGRAFÍA}

Cabreiros, A. (2011). La plataforma Moodle y la evaluación de los aprendizajes. Disponible en http:// www.uoc.edu/symposia/dret_tic2011/pdf/4.cebreiros_alvarez_eduardo.pdf

Fonseca, D.; Medrano, C. \& Orozco, V. (2013). Revista de Informática Educativa. Disponible en https:// revistainformaticaeducativa.unan.edu.ni/index.php/ie/article/view/19/10

Murillo, C. (2012). Ensayo"GeneralidadesdeMoodle". Disponibleenhttps://maestriainformaticaeducativa. wordpress.com/2012/11/08/ensayo-sobre-moodle/

Silva Quiroz, J. (2011). Diseño y moderación de entornos virtuales de aprendizaje (EVA). Barcelona: UOC. Disponible en http://educoas.org/portal/la_educacion_digital/146/publicaciones/entornos. html

UNAN-Managua. (2015). Plan Estratégico Institucional 2015-2019. UNAN Managua. Managua, Nicaragua 